\title{
Correction to: The presenting dental status of solid tumours with bone metastases requiring bone-targeting agents - part 1: an overview
}

The original article can be found online at https://doi.org/10.1038/s41415-021-3825-y

Journal's correction note:

Due to a production error, when the article 'The presenting dental status of solid tumours with bone metastases requiring bone-targeting agents - part 1: an overview' written by Vinod Patel, Sanford Grossman, Rana Wali et al. was originally published, the publication date on the publisher's internal portal read 14 January 2022. This has been corrected to 13 January 2022, the date the paper was first published online.

The journal apologises for any inconvenience caused. 